\title{
Changing scientific meetings for the better
}

\author{
Conferences are a pivotal part of the scientific enterprise, but large in-person meetings have several disadvantages. \\ As the pandemic experience has shown, online meetings are a viable alternative. Accelerating efforts to improve \\ conferences in virtual formats can lead to a more equitable and sustainable conference culture.
}

\section{Sarvenaz Sarabipour, Aziz Khan, Yu Fen Samantha Seah, Aneth D. Mwakilili, Fiona N. Mumoki, Pablo J. Sáez, Benjamin Schwessinger, Humberto J. Debat and Tomislav Mestrovic}

$\mathrm{S}$ cientific conferences are important avenues for researchers to share and discuss research findings, to exchange ideas and insights, and to network for collaboration and career development. Organizing inclusive and useful scientific meetings is a significant responsibility shared by researchers, scientific societies, and other organizations worldwide. Prior to 2020 , most conferences were held in-person and provided limited attendance opportunities for many researchers from diverse socioeconomic backgrounds, particularly early career researchers (ECRs), researchers from low- and middle-income countries, and junior principal investigators $(\mathrm{PIs})^{1}$. Some conferences in select disciplines have implemented valuable changes for the community, becoming more receptive to attendees with families. Due to the COVID-19 pandemic, a major shift has occurred towards virtual conferencing formats, triggering discussions in the scientific community on the current and future formats and organization of conferences. Here we provide recommendations for improving scientific meetings towards more efficient, sustainable, and equitable gatherings.

\section{The many disadvantages of in-person conferences}

We examined 270 national and international academic conferences held in person during 2018-2019 in various disciplines for their features, costs, and impact on the community. We found that many meetings could be improved significantly in terms of diversity, inclusivity, ECR promotion, networking and career development, venue accessibility, and, importantly, reducing the meetings' carbon footprint (Fig. 1 and Supplementary Information).

Carbon dioxide emissions. The increasing number and size of scientific conferences held in-person contribute to rising atmospheric carbon dioxide $\left(\mathrm{CO}_{2}\right)$ levels and thus to climate change. There are over 8.4 million full-time and full-time-equivalent researchers globally as of 2015, representing a growth of over $20 \%$ since 2007 . With the current career norms, researchers are expected to fly nationally and internationally, often several times a year, for scientific meetings. Conference attendance accounts for $35 \%$ of a researcher's footprint ${ }^{2}$. In hosting hundreds to thousands of researchers, conferences produce substantial air-travel-related $\mathrm{CO}_{2}$ emissions, comparable to the global annual per capita $\mathrm{CO}_{2}$ emissions in many countries, as well as large amounts of other waste in the form of promotional items. The overwhelming majority of conferences are not environmentally sustainable and lack clear green strategies or climate policies. Clearly, the scientific community that is supposed to understand the problem better should contribute to reducing their travel-related $\mathrm{CO}_{2}$ emissions ${ }^{3,4}$.

Attendance fees. The costs associated with attending conferences include meeting registration fees, airfares, accommodations, ground transportation, food, and event tickets. The registration fees alone often range from a few hundred to over a thousand US dollars. The total cost of attending large national and international scientific meetings is in the thousands of dollars, equivalent to one or more months of graduate and postdoctoral researcher net salary worldwide. As a result, a principal investigator may attend multiple conferences a year, but will have to budget thousands of dollars to send multiple trainees to one conference per year.

Visa requirements. Obtaining short-term visas to travel to scientific meetings is a major hurdle for many researchers, particularly those from developing nations ${ }^{1}$. A number of countries have explicitly stated that certain nationalities are not welcome or will have to endure lengthy, uncertain, and costly procedures to obtain a visa. Ongoing or long-term conflicts as well as recent changes in the political climate at conference destinations (often major scientific hubs), such as travel bans, have added to these challenges. These obstacles not only deeply affect the lives and careers of scientists working in developing countries, but also citizens of countries with visa restrictions who live and perform research in global scientific research centres and wish to attend conferences elsewhere

Inequity in access and means. The organization of many conferences leads to practices that exclude researchers on a wide range of factors including, but not limited to, gender, ethnic, racial, socioeconomic, health, and geographical backgrounds, as well as career stages. For instance, women and other under-represented researchers are the least likely to be offered opportunities to speak at or chair meetings in their discipline $e^{5,6}$. The current lack of diversity hinders advancement in scholarship, and it is especially discouraging to junior researchers. There has also been inertia in setting up and implementing more family-friendly policies at scientific meetings ${ }^{7}$. A small fraction of conferences includes some form of career development workshop and/or promotion events such as special symposiums, podium talks, and poster or oral presentation awards for ECRs.

How to improve academic conferences
Organize national and international
meetings in fully or mostly virtual
formats. Researchers, scientific societies,
and funders should work together to
improve the format and organization
of conferences (Fig. 2 and Table 1).
Conferences in remote vacation destinations
are difficult to justify, as these accumulate
a large carbon footprint, often without
clear scientific objectives and little to no
benefit to the local scientific community.
On the other hand, most ground-based
transportation modes produce lower $\mathrm{CO}_{2}$
emissions. Regional society meetings
provide many benefits, such as low hosting
costs, and they can be livestreamed,
recorded, and made available online for
the benefit of other researchers globally.
Regional conferences held in more
economic and public venues, such as

How to improve academic conferences and funders should work together to improve the format and organization of conferences (Fig. 2 and Table 1). Conferences in remote vacation destinations are difficult to justify, as these accumulate a large carbon footprint, often without clear scientific objectives and little to no benefit to the local scientific community. On the other hand, most ground-based transportation modes produce lower $\mathrm{CO}_{2}$ emissions. Regional society meetings provide many benefits, such as low hosting recorded, and made available online for Regional conferences held in more conomic and public venues, such as 


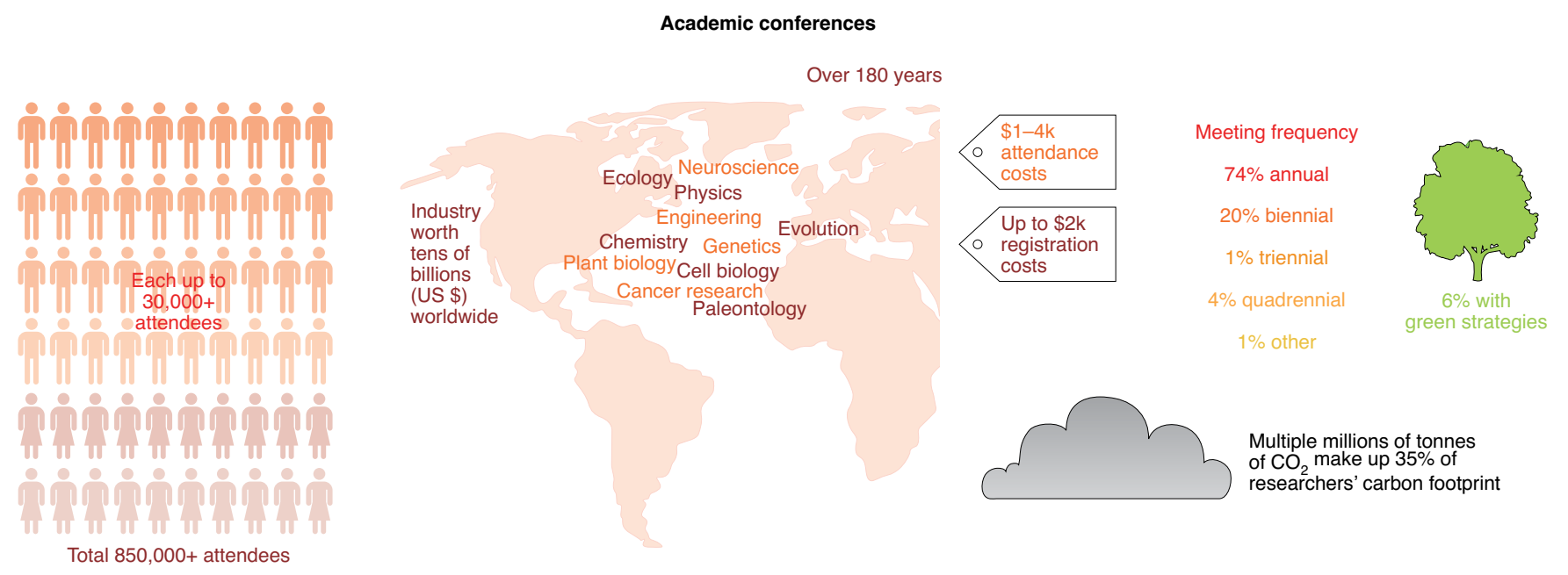

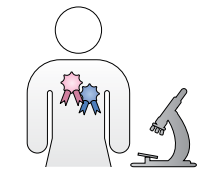

$38 \%$ promote ECRs with research awards

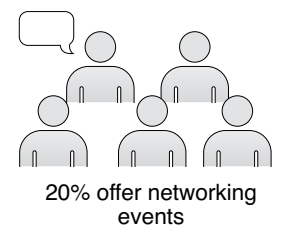
events
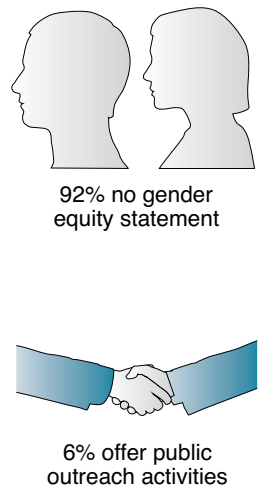
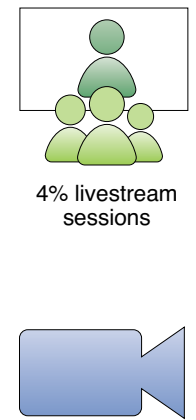

$11 \%$ offer eposters or video, audio digital libraries of recorded talks

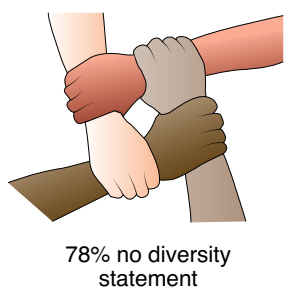

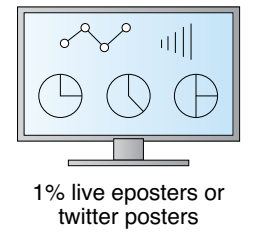

twitter posters

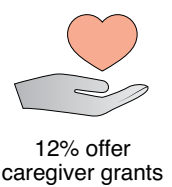

childcare services

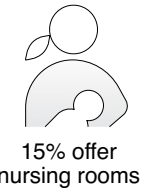

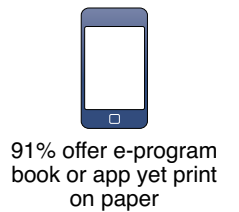
on paper

\author{
Promoting diversity \\ Grant writing Publishing \\ Data management Implicit bias \\ Peer-review training Ethics \\ Work-life balance Reproducibility \\ Mentoring Effective teaching \\ Science communication Public policy \\ Early-career faculty training \\ Succeeding in collaborations
}

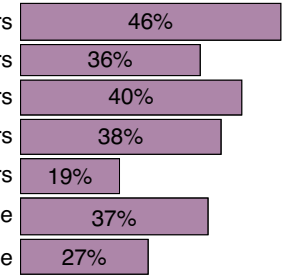

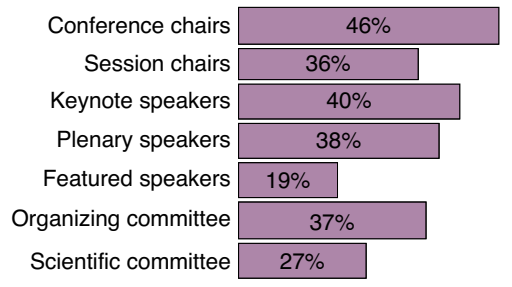

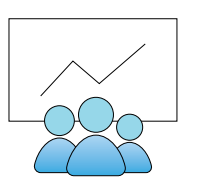

only $35 \%$ offer a career development workshop
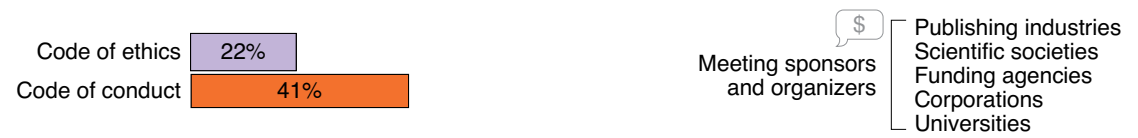

Fig. 1 | In-person academic conferences are costly and exclusive. Summary analysis of features of 270 conferences held in-person by over 150 scientific societies and organizations during 2018-2019 in various scientific disciplines (Supplementary Information). Aggregate analysis is presented from a database of in-person conferences (https://elifeambassadors.github.io/improving-conferences/). Overall, the majority of meetings were held frequently (annually), and their formats and organization lacked environmental sustainability, inclusive practices, and promotion and training opportunities for early-career researchers.

schools or university campuses, may also offer attendees the opportunity to visit local laboratories, tour facilities, and interact with the local scientists, potentially bringing benefit to the local community. These meetings enable interactions and discussions of science in ways that provide ECRs with ample opportunities to exchange ideas and meet established researchers.
Virtual global conferences and local hubs. Face-to-face interactions at conferences that are important in generating new ideas, networks, and collaborations can also be facilitated via digital conferencing. Reducing academic air travel does not impact professional success ${ }^{8}$. Speakers spend time and resources preparing talks for in-person conferences that end up reaching a limited audience with limited geographic impact, often restricted to wealthy economies. Switching to fully virtual conference modes will increase reach and access to knowledge worldwide. Multi-location in-person conferences, where participants only travel to a nearby location to interact with other 'local' scientists, are also viable ${ }^{9,10}$. Each hub may hold in-person keynote and panel 
Key considerations for every academic conference
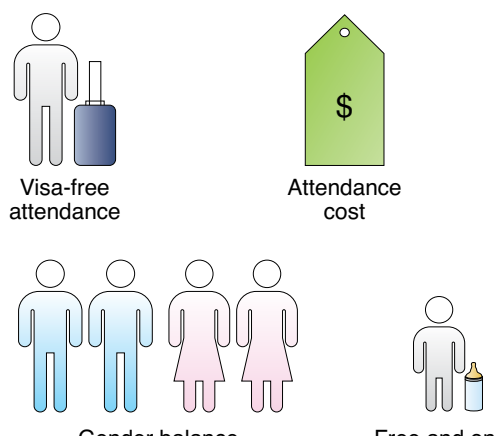

Free and on-site childcare

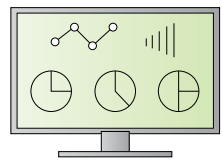

Electronic posters

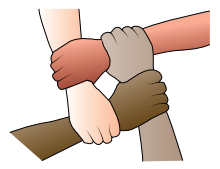

Diversity and inclusion statement

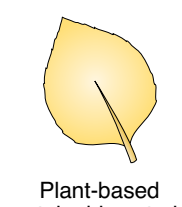

sustainable catering

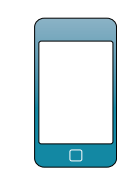

Electronic-only program books

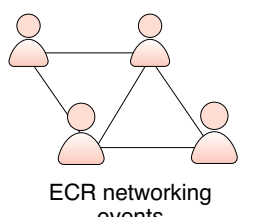

events
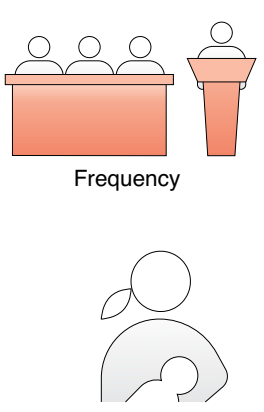

Nursing rooms

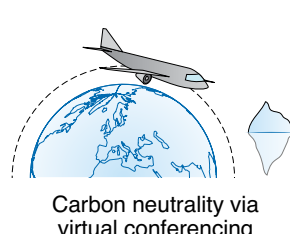

virtual conferencing
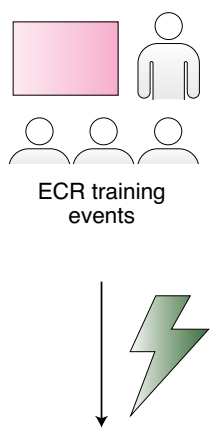

Reduce energy and resource use
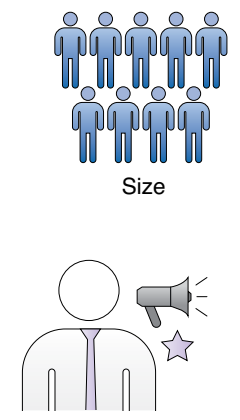

ECR promotion events

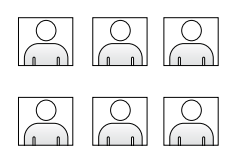

All talks livestreamed and recorded

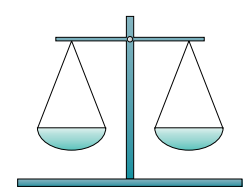

Code of ethics

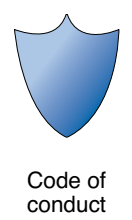

Fig. 2 | Key considerations for attendees and organizers of academic conferences. A checklist of recommended key considerations for improving conferences in hybrid or fully virtual formats for the benefit of all researchers worldwide.

sessions, but the hubs can also be connected for virtual panel sessions and discussions (broadcast digitally across continents); such an arrangement enables speakers to present talks and posters remotely. Livestreaming allows chat amongst other online attendees in real time, access to sessions that fit attendee schedules, and the ability to stream sessions from any devices, while creating more space for researchers to interact and connect. The number of conferences that are livestreamed or held in a virtual reality setting has increased over the past few years, particularly in 2020 due to the COVID-19 outbreak, showing great success ${ }^{11}$.

Foster digital networking by investing in relevant, immersive, and interactive experiences. Organizers of virtual conferences must develop strategies for facilitating digital connections. Fully streaming conferences (beyond video-conferencing select talks) and scientific electronic panels can be made feasible without many significant additional resources, accompanied by online discussions and Twitter (poster) conferences. Participants can also record and upload videos of their presentations that are posted to the meeting website. During several weeks when the conference is 'open', attendees can watch the videos, ask questions, and interact with the speakers on online forums; educational and hiring opportunities can be offered and applied for. Slack groups have taken the initiative to connect researchers within and across disciplines globally, via group-based and one-on-one online discussions and keep them virtually connected afterwards.
Electronic (online) poster sessions can provide each presenter with the opportunity to tag their poster with a 3-5-min talk summarising their work. Attendees watching video presentations of talks and posters can direct-message the presenting authors for inquiries via email or the online meeting platform. Digital discussion forums can serve to assist communication between early-career and senior researchers, as writing a comment or question in a forum can feel less intimidating than approaching an established scholar in person. With more time to think the presentations through, attendees can put their best foot forward during interactions and networking with speakers.

Incentives and mandates to support and promote low-carbon research careers. Prior to the COVID-19 pandemic, business-related air travel by academics at universities continued to grow, reaching tens of millions of miles flown in one year. Institutional practices should shift to mandate severe reductions in academic air travel, as these changes will significantly reduce the academic carbon footprint. Purchasing carbon offsets does not reverse the damaging impact of researchers flying on climate and living ecosystems, and the scale of the efforts and cost to actually offset emissions may be prohibitively high. Institutions and funders can implement a carbon tax on air travel emissions caused by academic flying and mandate traveling by train for short travel time. Funds from a carbon tax can be used to fund research that could fight against the climate crisis.

As researchers re-evaluate their practices, it is also essential that academic institutions, scientific societies, and funding agencies coordinate and mandate sustainability codes of conduct and funding to only organize academic conferences in fully virtual and multihub, hybrid in-person/virtual formats, simultaneously increasing accessibility for all ${ }^{9,10}$. At many institutions and disciplines, scholars are granted tenure and promotions based in part on the number of their research presentations at professional conferences. Furthermore, as postdoctoral and tenure-track jobs are scarce in many academic disciplines, ECRs face mounting pressure to attend conferences to network with potential colleagues. Changes can also be expected in funding and promotion requirements so that academics on tenure-track do not have to choose between delivering an international talk and receiving a grant or tenure promotion. The proposed changes below will in turn encourage a culture of academic research that is more equitable and less reliant on traveling. 


\section{Table 1| Actions to improve small and large, hybrid and fully virtual conferences}

Open-access dissemination of conference materials

Require open-access deposition of conference submissions and materials on public repositories to improve utility, transparency, accreditation, and citation.

Increasing accessibility for researchers with disabilities

Provide closed captions and offer transcripts ${ }^{15}$, as well as text-reader-friendly documents and audio-described versions of presentations.

\section{Assisting attendees with internet connectivity or financial challenges}

Eliminating merchandise

Reducing plastic usage

Including green events

Organizing public outreach activities for attendees

Improving attendee physical safety

Improving trainee \& ECR faculty career development

Improving equity in chairs and speaker selection criteria

Organizing useful networking activities

Creating databases for scientific conferences
Provide internet hotspots to enable virtual participation for attendees in remote geographic locations, and help participants experiencing financial difficulties with sourcing local sponsors for support.

Source compostable conference name badges, lanyards, and gifts or exclude these altogether and repurpose the souvenir costs to promote ECRs.

Encourage attendees to bring their own reusable materials and to take notes electronically on their portable devices.

Retire disposable cups and cutlery during refreshment breaks and use compostable or reusable dishware when serving food.

Arrange for environmental improvement events such as forest or beach clean-up walks, helping attendees to socialize and discuss science while helping the planet.

Arrange for interested conference attendees to offer public lectures at local schools and libraries with a layperson version of their presentation.

Organize safe transportation modes and accommodation, implement anti-harassment policies, a clear system for reporting and handling such issues, and mandatory safety apps or a buddy system for attendees to accompany or warn each other and report safety concerns.

Organize training workshops and implement special ECR symposia, poster sessions, and awards to highlight women and other under-represented groups and to advocate for up-and-coming ECRs entering the job market.

Organize every session with at least one ECR chairing and organizing the $Q \& A$ discussions; specifically invite trainees to ask questions or take the first questions from ECRs.

Organize networking events and random groupings at breakout rooms to connect researchers across career stages and disciplines and encourage discussions and planning for collaborations or seeking mentorship.

Report conference attendee and speaker gender, geographic, and career-stage statistics online; create a central database of key sustainability and equity considerations of conferences, enabling researchers to identify the most beneficial gatherings.
Incentives and mandates to improve intersectionality and career-stage equity at conferences. Equity in planning scientific meetings creates fair opportunities for all, and inclusivity creates a welcoming environment for all participants. Conference organizers must make conscious efforts to maintain fair representation of all researchers.

Maintaining gender balance impacts both speakers and ECRs, who need to see representation and role models of their own gender in their field. Without clear guidelines and targets, unreasonable excuses are made for lack of diversity. Organizers should publish a statement of equity, diversity, and inclusion and should actually achieve inclusion of $50 \%$ or higher female speakers. The process by which speakers are selected from abstract submissions can be improved by removing intended and unintended biases.

There has been an increase in the number of minority researchers in many scientific disciplines across graduate, postdoctoral, and faculty career stages, yet this is often not reflected in the composition of conference programmes $^{12}$. Blinding the selection of talks, removing conflicts of interest, using open databases listing ECRs from under-represented groups, and planning fewer long talks in favour of more short talks may all help ensure that minority groups of scientists are welcomed and equally represented ${ }^{13,14}$.
Funders and meeting organizers need to also pledge and enforce gender and career-stage diversity and equity policies as well as public inclusion declarations and guidelines, and they should publish the names of speakers and session chairs in advance of the conference program. Gender and career-stage disparities also exist in participation during discussions, as these most often are led by professors. Since the number of ECRs has increased globally, a number of talks can be allocated to early- and mid-career researchers. Senior faculty can nominate an ECR trainee or recommend an ECR colleague in their place to attend the conference.

If the current widespread digitization of conferences continues beyond the pandemic, global access to knowledge will increase substantially during and after meetings, enabling greater democratisation of science, and equalising differences between researchers of diverse means and backgrounds. Concerted efforts in organization are needed alongside new conferencing formats to ensure scientific meetings improve for researchers worldwide.

\section{Sarvenaz Sarabipour (D) $1{ }^{凶}$, Aziz Khan (iD) 2,3, Yu Fen Samantha Seah (D), \\ Aneth D. Mwakilili (D)5,6, Fiona N. Mumoki (D)7, Pablo J. Sáez (D) 8,9 \\ Benjamin Schwessinger (iD) 10 , Humberto J. Debat (DD ${ }^{11}$ and Tomislav Mestrovic (D) 12,13}

${ }^{1}$ Institute for Computational Medicine \& Department of Biomedical Engineering, Johns Hopkins University, Baltimore, MD, USA. ${ }^{2}$ Centre for Molecular Medicine Norway (NCMM), Nordic EMBL Partnership, University of Oslo, Oslo, Norway. ${ }^{3}$ Stanford Cancer Institute, Stanford University School of Medicine, Stanford University, Stanford, CA, USA. ${ }^{4}$ European Molecular Biology Laboratory, Genome Biology Unit, Heidelberg, Germany. ${ }^{5}$ Swedish University of Agricultural Sciences, Plant Protection Department, Alnarp, Sweden. ${ }^{6}$ University of Dar es Salaam, Department of Molecular Biology and Biotechnology, Dar es Salaam, Tanzania. ${ }^{7}$ Department of Zoology and Entomology, University of Pretoria, Pretoria, South Africa. ${ }^{8}$ Institut Curie, Paris Sciences \& Lettres Research University, CNRS, UMR 144, Paris, France. ${ }^{9}$ Department of Biochemistry and Molecular Cell Biology, University Medical Center Hamburg-Eppendorf, Hamburg, Germany. ${ }^{10}$ Research School Biology, The Australian National University, Acton, Australian Capitol Territory, Australia. ${ }^{11}$ Center of Agronomic Research, National Institute of Agricultural Technology (IPAVE-CIAP-INTA), Córdoba, Argentina. ${ }^{12}$ Clinical Microbiology and Parasitology Unit, Dr Zora Profozic Polyclinic, Zagreb, Croatia. ${ }^{13}$ University Centre Varazdin, University North, Varazdin, Croatia.

$凶_{e-m a i l: s s a r a b i 2 @ j h u . e d u}$ 
Published online: 15 March 2021

https://doi.org/10.1038/s41562-021-01067-y

\section{References}

1. Waruru, M. Nature https://doi.org/10.1038/d41586-018-06750-1 (2018).

2. Achten, W. M. J., Almeida, J. \& Muys, B. Ecol. Indic. 34, 352-355 (2013).

3. Sanz-Cobena, A. et al. Nat. Food. 1, 187-189 (2020).

4. Nathans, J. \& Sterling, P. eLife 5, el5928 (2016).

5. Larson, A. R. et al. J. Womens Health (Larchmt.) 29, 550-560 (2020).

6. Else, H. Nature 573, 184-186 (2019).
7. Langin, K. Science https://doi.org/10.1126/science.caredit. aaw3741 (2018).

8. Wynes, S., Donner, S. D., Tannason, S. \& Nabors, N. J. Clean. Prod. 226, 959-967 (2019).

9. Coroama, V. C., Hilty, L. M. \& Birtel, M. Telemat. Inform. 29, 362-374 (2011).

10. Abbott, A. Nature 577, 13 (2020).

11. Reshef, O. et al. Nat. Rev. Mater. 5, 253-256 (2020).

12. Carter, A. J., Croft, A., Lukas, D. \& Sandstrom, G. M. PLoS One 14, e0212146 (2019).

13. Martin, J. L. PLOS Comput. Biol. 10, e1003903 (2014).

14. Vallence, A. M., Hinder, M. R. \& Fujiyama, H. PLoS One 14, e0220481 (2019).

15. Gernsbacher, M. A. Policy Insights Behav. Brain Sci. 2, 195-202 (2015).
Acknowledgements

The authors thank R. Sever, R. D. Vale, A. W. Bisson Filho,

D. Trépel, E. Emmott, R. Kudva, S. J. Burgess, J. D. Fernandes,

A. Tay, V. Ilangovan, and I. Lam for valuable comments on this work.

Competing interests

The authors declare no competing interests.

Additional information

Supplementary information The online version

contains supplementary material available at https://doi.

org/10.1038/s41562-021-01067-y. 